\title{
Exploring and Measuring Possible Co-Existences between DVB-T2- Lite and LTE Systems in Ideal and Portable Fading Channels
}

\author{
L. Polak ${ }^{*}$, O. Kaller, L. Klozar, J. Sebesta and T. Kratochvil \\ Sensor, Information and Communication Systems (SIX) \\ Research Center \\ Department of Radio Electronics, Brno \\ University of Technology \\ Brno, Czech Republic \\ *polakl@feec.vutbr.cz
}

\begin{abstract}
From the point of technical innovations the development and standardization of Digital Video Broadcasting $-2^{\text {nd }}$ Generation Terrestrial (DVB-T2) and Long-Term Evolution (LTE) systems are definitely the most significant results in the last decade. These systems have a very high potential to fulfill the highest user requirements, but they can operate in the same frequency spectrum. As a result, different co-existence scenarios can occur. In this paper, we explore and measure the co-existence between DVB-T2-Lite (e.g. portable TV) and LTE multimedia services in ideal and portable fading channel models. Theoretical backgrounds of the investigated co-existence scenarios, proposal and realization of an appropriate workplace for their measuring and evaluation are presented and described. Moreover, deeper investigation of the mutual influence of the DVB-T2 system on the LTE one is also explored and graphically illustrated. The obtained results show that these co-existences could be critical for both systems from the point of providing multimedia services with a constant level of Quality of Services (QoS).
\end{abstract}

Keywords: DVB-T2-Lite, LTE, Co-existence of wireless systems, portable fading channels, SDR, BER, EVM, MER.

\section{Introduction}

The use of advanced wireless and mobile networks has expanded into the daily life of people. They can provide many useful services which people use every day and their life without these services is unimaginable. However, demands from users on these services are higher and higher. The concept "access to anyone, anywhere, at any time" is nowadays the main target of each mobile and broadcast system. Hence, increasing demand for steady wireless multimedia services is a key feature of modern markets. Consequently, research for next generation wireless communication standards is focused on the development of robust, but also effective transmission systems which can operate in a high range of the frequency spectrum [1]-[3].

To fulfill these requirements, on the last World Radio Conference (WRC-2007) it was decided to allocate the $790 \div 862 \mathrm{MHz}$ frequency band to mobile services in Europe as from 2015, and allowed several Europe regions before 2015. However, from a technical and market perspective, this decision will give rise to creating new interference scenarios and co-existence between two types of services, using the same or adjacent frequency bands. In our concrete case, these two services should be the newest mobile (e.g. LTE) and TV broadcast services (DVB-T2) [1], [4], [5].

Based on recent research results and a set of commercial requirements, the Digital Video Broadcasting (DVB) consortium has successfully developed the second generation of satellite (DVB-S), cable (DVB-C) and terrestrial (DVB-T) standards, marked as DVB-S2/C2/T2. From the point of features, the DVB-T2 technology will be the most used DVB standard that could provide increased capacity and robustness in the terrestrial environment, mainly for high definition TV (HDTV) broadcasting. Moreover, within the DVB-T2 standard a new profile DVB-T2-Lite has been developed. It allows simple implementations of the receiver for low capacity applications, like mobile or portable TV broadcasting. It is based on the same core of technology as the DVB-T2 standard, 
but only uses a limited number of available modes. More precisely, it avoids modes which require the most complexity and memory and allows more efficient receiver designs (power consumption, smaller silicon size) to be used. More technical details and recommendations for the DVB-T2-Lite profile can be found in [6]-[8]. DVB-T2/T2-Lite services, such as DVB-T, are operating within the existing VHF $(174 \div 230 \mathrm{MHz})$ and UHF $(470 \div 870$ $\mathrm{MHz}$ ) spectrum.

The development in the field of mobile communications is also rapidly increasing. The goal of this development is to increase the capacity and speed of wireless data networks (its redesign and simplification), using new techniques and modulations. The result is a very perspective LongTerm Evolution (LTE) system that will definitely replace the current GSM/UMTS standards in the future. The LTE, as defined by the 3GPP (3rd Generation Partnership Project), is a very flexible radio interface that offers a high scale of adjustable system parameters [9]-[11], higher than also very perspective High Speed Packet Assess (HSPA) [3]. The LTE services can be operated in the frequency bands that are already available for existing $3 \mathrm{G}$ networks $(880 \div 960 \mathrm{MHz})$. Moreover, additional ranges $(2.5 \div 2.7 \mathrm{GHz})$, and frequencies $(791 \div 821 \mathrm{MHz})$, are allocated for usage [4].

Thanks to significant technical innovations DVBT2-Lite and LTE systems have a great potential to give wireless multimedia services in high quality. However, both of them can work in the same frequency bands [4]. Hence, there are possible different co-existence and unaware interference scenarios. The focus of this paper is to explore and measure the possible co-existences between DVBT2-Lite and LTE services and their impact on the quality (on the physical layer) of both services. Moreover, in our measurement we will consider not only an ideal channel environment, but also portable fading channel conditions.

The rest of this paper is organized as follows. After the introduction, the state-of-the-art in this field is presented in Section 2. The explored co-existence scenarios, considered portable fading channel models, and used system parameters are outlined in Section 3. This section also contains a brief description of our proposed and realized workplace and method for measuring interactions between the explored mobile services. Section 4 contains the evaluation and discussion of the results, obtained from our measurements. Finally, the paper concludes in Section 5.

\section{Background and related works}

Unwanted co-existence scenarios between different wireless systems which work in the same or adjacent frequency spectrum is not a new phenomenon [12]-[14]. The impact of the coexistence and interferences between different wireless communication services on the capacity and Quality of Services (QoS) is still being explored today. The topicality of this issue is evidenced by a lot of studies and research.

In literature many works can be found which deal with this topic and, in general, they can be divided into 2 main groups. First group of these works focuses on the investigation of the co-existence and adjacent channel interferences between different but same kind of wireless systems, e.g. mobile systems and networks [15]. In [16] authors proved that in advanced mobile networks between femto cells which share common frequency spectrum with macro cells so-called cross-tier interferences can occur. Brief study of intra/inter interferences which may occur from the coexistence between Worldwide Interoperability for Microwave Access (WiMAX) and LTE at uplink were presented in [17]. The common result of the mentioned studies was that these mutual interferences [18] can decrease the quality and the capacity of the considered $3 \mathrm{G} / 4 \mathrm{G}$ mobile networks (GSM, UMTS, WIMAX and LTE).

The second group includes studies which deal with investigation, modeling, simulating and measuring of interference scenarios, occurring between different communication standards [19], e.g. DVB and mobile system. In [1], [5], [20], [21] different types of interferences (e.g. blocking interference, spurious emissions interference and adjacent channel interference) are investigated, not only on DVB-T/H (Terrestrial/Handheld), but between DVB$\mathrm{T} / \mathrm{H}$ and other types of wireless services operating in the UHF frequency band. Furthermore, authors in [20] and [22]-[24] deal with possible crossborder interferences which can occur when UMTS and LTE mobile systems interfering into DVB-T broadcasting system, respectively. 
As can be seen from presented references, exploring interferences, as a product of different co-existence scenarios of different multimedia technologies, is a perspective and hot topic. In our last works [25], [26], we explored the influence of mobile network interfering products on DVB-T/H broadcasting services. In this extended paper (based on [27]), we focus on measuring interactions between DVB-T2Lite and LTE services. Both of these services are potential candidates to provide multimedia services in high quality for mobile terminals and both of them can be operated in the same frequency range.

\section{Explored co-existence scenarios and proposed experimental measurement}

As it was mentioned above, we are mainly focusing on the co-channel scenarios where the wanted (useful) and the unwanted (interfering) signals are located in the same frequency band. In this part, behind the outlined co-existence scenarios, the proposed and realized workplace and the measurement setup are introduced.

\subsection{Conception and analyzed co-existence scenarios}

We consider a co-existence scenario, when an LTE base transceiver station (BTS), transmitting a downlink signal, acts as an interferer on the digital TV (DTV) receiver and vice versa. The general scenario is clearly illustrated in Fig. 1. We have a common cell for DVB-T2-Lite and LTE services. The owner of a tablet is receiving DVB-T2-Lite services at a frequency of $794 \mathrm{MHz}$. At the same time, another user of a smartphone is receiving LTE services, provided from a mobile operator at a frequency of $802.2 \mathrm{MHz}$. In the case, when the bandwidth of the LTE signal is $10 \mathrm{MHz}$, then it can interfere with the upper spectrum side of the T2Lite signal (from 794 to $798 \mathrm{MHz}$ ). It means visible artifacts in the DTV reception or complete failure to receive the wanted (DVB-T2-Lite) signal. Of course, the level of the impact of occurred interferences depends on the level of the unwanted signal. Other possible co-existence scenarios, which are considered in this work, are plotted in Fig. 2.

\subsection{Considered fading channel models}

In mobile/terrestrial wireless communications, the transmitted radio waves often do not reach the receiving antenna directly. In real terrestrial transmission scenarios, the line-of-sight (LOS) path is always affected by different obstacles (e.g. trees, hills, buildings, moving cars). Distribution of the DVB-T2-Lite and LTE mobile multimedia services by way of terrestrial transmitters is the natural technology of broadcasting. The received signal should be interpreted as the overall effect, the sum of various influences created by noise, interference and Doppler shift and type of distribution (spectrum) [28].

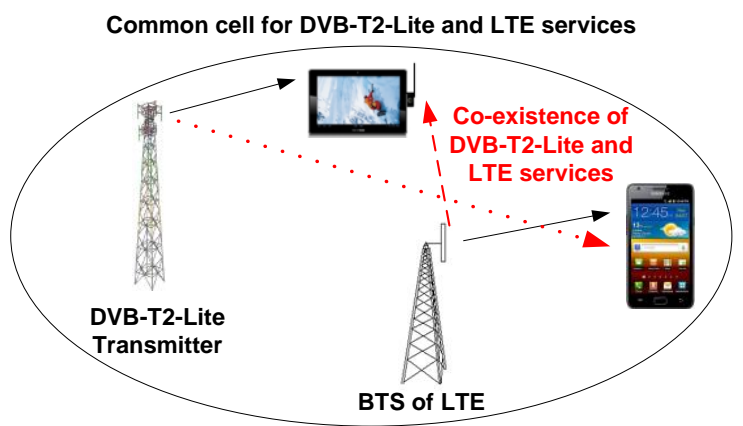

Figure 1. Possible co-existence scenario, when DVB-T2-Lite and LTE services are operated in the same frequency band.

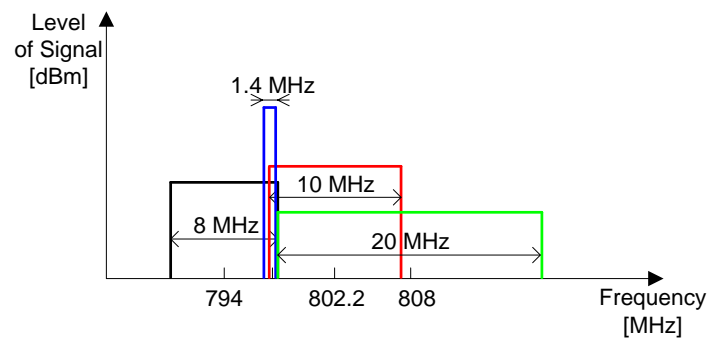

Figure 2. Spectrum of analyzed co-existence scenarios between DVB-T2-Lite (black color) and LTE (blue, red and green colors) services.

In this paper we will investigate the above described co-existence scenarios in the portable fading channel too. We considered that both T2Lite and LTE services are transmitted/received in a pedestrian indoor environment. Therefore, in our experiments we used pedestrian indoor (PI) and extended pedestrian $A(E P A 5 \mathrm{~Hz}$ ) fading channel models, respectively.

The PI channel model has been developed by the Wing-TV project for describing slowly moving (at a 
speed approx. $3 \mathrm{~km} / \mathrm{h}$ ) handheld indoor TV reception [28]. This channel model is based on measurements in the DVB-T/H single frequency network (SFN) and has paths from two different transmitter locations. The PI channel consists of 12 independent paths. The first path has Rice-Gauss and the remaining eleven ones have a RayleighGauss Doppler spectrum [29]. When the working frequency is $794 \mathrm{MHz}$, then the maximal Doppler shift is approx. equal to $2.2 \mathrm{~Hz}$.

Particularly, in the LTE system, the EPA channel model is used to model the reference environment characterized by a low delay spread [30]. The main parameters of this model are specified in [31]. The EPA channel consists of 7 independent paths. All the taps have a Rayleigh-Jakes Doppler spectrum. In addition to a multipath delay profile, the maximum Doppler frequency is specified for each multipath fading propagation condition. In our case it is $5 \mathrm{~Hz}$. Impulse response of both fading channel models are plotted in Fig. 3 and Fig. 4.

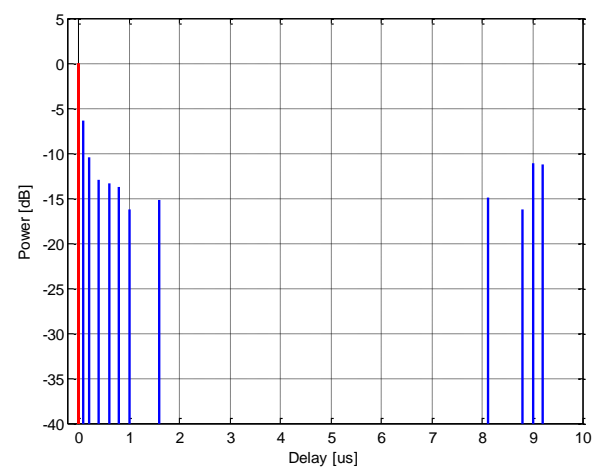

Figure 3. Impulse response of the PI channel.

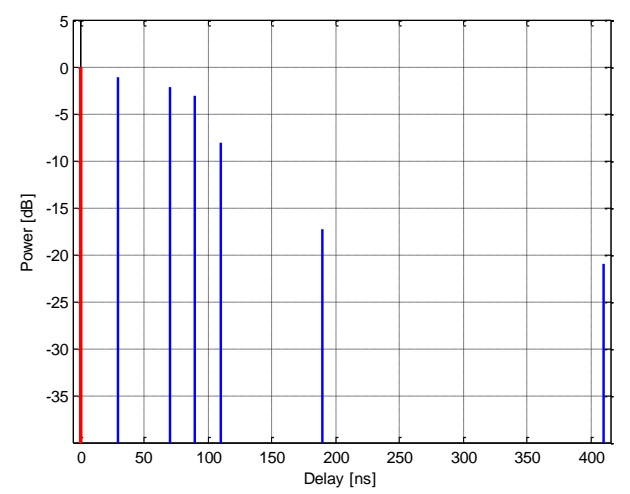

Figure 4. Impulse response of the EPA $5 \mathrm{~Hz}$ channel.

\subsection{Measuring setup and principle of the measuring}

Our purpose is to measure the impact of the interfering LTE services on the degradation of performance of the DVB-T2-Lite ones and vice versa when these services are operating in the same frequency band. The proposed general block diagram of the realized measurement of coexistences between both mobile services is shown in Fig. 5. Based on this conception, a laboratory workplace (in the Laboratory of Mobile Communication Systems, Brno University of Technology) was realized with appropriate measurement equipment (see Fig. 6), supported by the SIX research center [32].

The basic principle of our measurement method is as follows. In our case, the interfered DVB-T2Lite signal is generated at a frequency of 794 $\mathrm{MHz}$. It has a classic $8 \mathrm{MHz}$ bandwidth, works in $2 \mathrm{~K}$ orthogonal frequency division multiplexing (OFDM) and uses 16QAM inner non-rotated modulation. The measuring technique consists of keeping a constant level of T2-Lite signal and increasing the level of the interfering signal. We set the level of the DVB-T2 signal at a value of $55.8 \mathrm{dBm}$ [33].

The generated LTE services, which negatively affect broadcasted mobile TV services, operate at frequencies from $791 \mathrm{MHz}$ to $821 \mathrm{MHz}$ [3]. In this frequency spectrum, LTE transmits in the downlink using frequency-division duplexing (FDD) duplex mode. The LTE signals, which interact with DVB-T2-Lite mobile services, are produced in R\&S SMU200A. LTE uses QPSK, 16QAM and 64QAM modulation formats along with scalable channel bandwidths from $1.4 \mathrm{MHz}$ to $20 \mathrm{MHz}$. Hence, we have generated different LTE signals with different bandwidths and types of modulations. Ten subframes were generated, where the used modulation types were equally used (3XQPSK; 3x64QAM and 4x16QAM). The bandwidths of LTE signals were $1.4,10$, and $20 \mathrm{MHz}$, respectively.

After sufficient generation of both wireless services, they are combined and then the splitter is used for dividing both signals, which are measured with appropriate measuring devices (see Fig. 6). More detailed system settings, which were used for our measurement, are summarized in Table 1. 


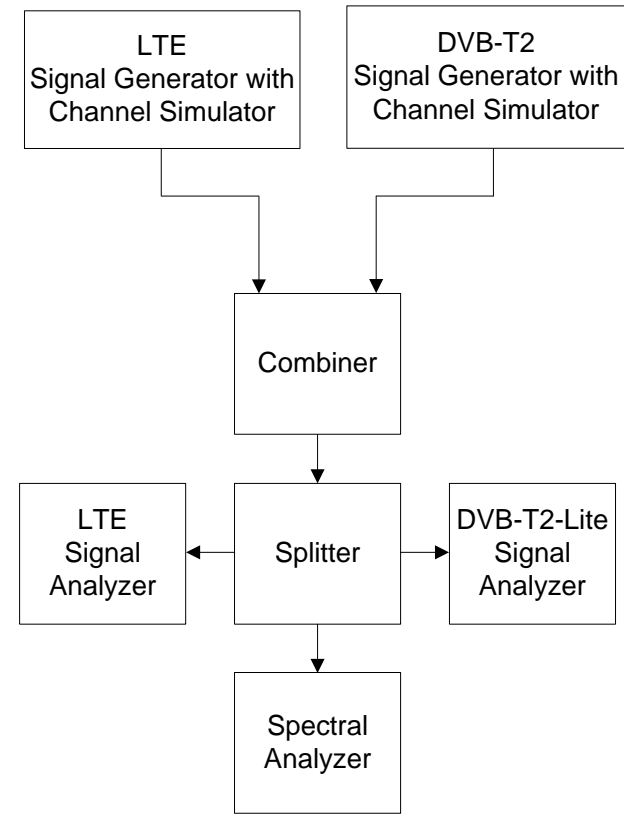

Figure 5. General block diagram of workplace for measuring the interaction between DVB-T2-Lite and LTE networks.

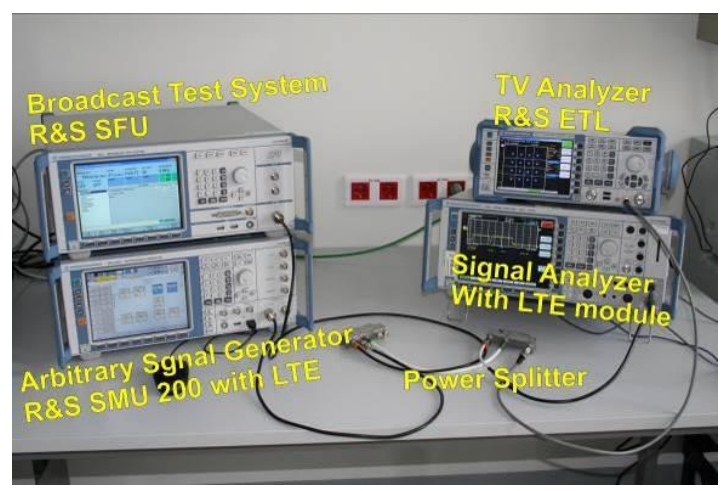

Figure 6. Realized laboratory workplace for measuring the interaction between DVB-T2-Lite and

LTE services (LTE generator, SFU DVB-T2-Lite transmitter, DVB-T2-Lite test receiver and analyzer and spectral analyzer).

\section{Measurement results and their evaluation}

To evaluate the QoS of the DVB-T2-Lite system we used two criterions. The first one is the classic Quasi Error-Free (QEF) operation [7], defined at BER after LDPC decoding less or equal to $1.10^{-}$. QEF is a minimal limit in DVB-T2 standard for achieving video service availability without noticeable pixelization in the video. The second criterion is based on the feature of the LDPC decoding. The performance of LDPC codes, and therefore the performance of DVB-T2-Lite, can be improved by increasing the number of decoding iterations. However, a higher number of decoding iterations has a larger impact on the power consumption of the user terminal. It is an important fact from the point of the mobile and portable TV reception. Therefore, we also focus on how the occurred co-existences influence the amount of repeated LDPC decoding, needed for successful achieving of QEF limit.

\begin{tabular}{|c|c|c|}
\hline Settings & DVB-T2-Lite & LTE \\
\hline $\begin{array}{l}\text { Code Rate } \\
\text { (CR) }\end{array}$ & $\begin{array}{c}2 / 3 \\
(\mathrm{LDPC}+\mathrm{BCH})\end{array}$ & $\begin{array}{c}1 / 3 \\
\text { (Turbo) }\end{array}$ \\
\hline $\begin{array}{l}\text { FFT Size/ } \\
\text { Channel } \\
\text { Bandwidth }\end{array}$ & 2048 (8 MHz) & $\begin{array}{c}128(1.4 \mathrm{MHz}) \\
1024(10 \mathrm{MHz}) \\
2048(20 \mathrm{MHz})\end{array}$ \\
\hline Modulation & 16QAM & $\begin{array}{l}\text { QPSK } \\
\text { 16QAM } \\
\text { 64QAM }\end{array}$ \\
\hline $\begin{array}{c}\text { Constellation } \\
\text { rotation }\end{array}$ & $\begin{array}{l}\text { no } \\
\text { yes }\end{array}$ & - \\
\hline Guard Interval & 56 us & 4.7 us \\
\hline $\begin{array}{c}\text { Transmission } \\
\text { Technique }\end{array}$ & $\begin{array}{c}\text { SISO } \\
\text { (Broadcasting) }\end{array}$ & $\begin{array}{c}\text { SISO } \\
\text { (Downlink) }\end{array}$ \\
\hline RF Level [dBm] & -55.8 & $-62.2 \div-50.9$ \\
\hline Frequency & $794 \mathrm{MHz}$ & 791 to $821 \mathrm{MHz}$ \\
\hline Channel/Band & C53 & Band 20 \\
\hline $\begin{array}{l}\text { Channel } \\
\text { Models }\end{array}$ & PI3 & EPA $5 \mathrm{~Hz}$ \\
\hline $\begin{array}{l}\text { Method of } \\
\text { Decoding }\end{array}$ & $\begin{array}{c}\text { LDPC } \\
\text { (hard decision) }\end{array}$ & Max Log-Map \\
\hline $\begin{array}{l}\text { Number of } \\
\text { Decoidng } \\
\text { Process }\end{array}$ & $\begin{array}{c}\text { automatically } \\
\text { depends on the } \\
\text { channel } \\
\text { conditions }\end{array}$ & $\begin{array}{c}\text { automatically } \\
\text { depends on the } \\
\text { channel } \\
\text { conditions }\end{array}$ \\
\hline
\end{tabular}

Table 1. Settings used for exploring the co-existence between DVB-T2-Lite and LTE services.

To evaluate the performance and QoS of the LTE system, EVM (Error Vector Magnitude) was used. In general, EVM is a measure used to quantify the performance of a communication system. In the area of LTE, it is a measurable vector in the IQ constellation diagram between the ideal constellation point and the point, received by the receiver. For each modulation, used in LTE, there is a defined EVM limit, for which the transmitted signal has an acceptable quality. This limit is equal 
to 17.5 for QPSK, 12.5 for 16QAM and 8.0 for 64QAM in [\%], respectively [9].

The EVM dependency of QPSK, 16QAM and 64QAM modulations on the frequency overlap of the payload carriers are shown in Fig. 7 a) and b). The expression "frequency overlap" defines the level of channel overlaps between the co-existing LTE and DVB-T2-Lite channels in $\mathrm{kHz}$ and "payload" is represents the useful data (carriers). The obtained results are related to spectral density ratio (SDR) ratio, equaling $6.5,-1.8$ and $5.0 \mathrm{~dB}$ when bandwidth of LTE signal is $1.4,10$ and $20 \mathrm{MHz}$, respectively. The SDR is defined as the power ratio between LTE and DVB-T2-Lite per unit of the used bandwidth. Its value is calculated as follows:

$\mathrm{SDR}=P_{\mathrm{LTE}}-10 \log B_{\mathrm{LTE}}-\left(P_{\mathrm{TV}}-10 \log B_{\mathrm{TV}}\right)$

where $P_{\mathrm{LTE}}$ is the power of the LTE signal, $B_{\mathrm{LTE}}$ expresses the bandwidth of the used LTE channel, $P_{\mathrm{TV}}$ is the power of DVB-T2 signal $(-55.8 \mathrm{dBm})$ and $B_{\mathrm{TV}}$ presents the bandwidth of the used TV channel $(8 \mathrm{MHz})$.

Firstly, all measurements were done at ideal channel conditions (see Fig. 7 a)). It means that the carrier-tonoise $(\mathrm{C} / \mathrm{N})$ ratio is higher than $40 \mathrm{~dB}$. From the obtained results, it is clearly seen that the level of the frequency overlap of the explored co-existing systems has a large impact on the availability of the modulations in the LTE system. Based on the minimal requirement [9], [30], the critical limit of EVM of the considered types of modulations (see bold black dashed lines in Fig. 7), at which the transmission in the specific sub-frames is without problems, is also dependent on the robustness of the type of modulation and used channel bandwidth. From this point of view, QPSK and 64QAM modulations have the highest and lowest resistance against frequency overlap, respectively. In general, the performance of transmission in each sub-frame, independently on the type of modulation used, fell down slowest and fastest when $B_{\mathrm{LTE}}$ is equal to $1.4 \mathrm{MHz}$ and $20 \mathrm{MHz}$. When we used an LTE system with a signal bandwidth of $20 \mathrm{MHz}$, then the values of EVM for all types of modulations were high. In the case when $B_{\mathrm{LTE}}=20 \mathrm{MHz}$ at $S D R=-5.0 \mathrm{~dB}$, the subframes, which use QPSK modulation, work without problem, when the frequency overlap is less than $1000 \mathrm{kHz}$.
Secondly, the same measurements were done for the above considered and briefly described channel models and the results are plotted in Fig. 7 b). All measurements were done with two channel environments. The Gaussian (AWGN) channel was used as a reference $(\mathrm{C} / \mathrm{N}=25 \mathrm{~dB})$. The PI (DVBT2-Lite) and EPA $5 \mathrm{HZ}$ (LTE) fading channel models were used as a second considered transmission environment. In the legend of Fig. 7 b) this fact is marked by the abbreviation " $\mathrm{FCH}$ " (fading channel). The $\mathrm{C} / \mathrm{N}$ ratio was equaled to $25 \mathrm{~dB}$ in all cases. The SDR is equal to $0.93 \mathrm{~dB}$ (the spectral density of the T2-Lite level is lower than the level of LTE services) and the $B_{\text {LTE }}$ was $10 \mathrm{MHz}$. Moreover, we explored the situation, during co-existence scenarios, when the power level of the LTE signal was less, equal or higher than that of the DVB-T2Lite signal.

As can be seen, the obtained results are significantly different when compared with results from the ideal channel environment. Thanks to higher delays and the Doppler spectrum features, the resistance of both communication systems to the noises during co-existence is much less. For example data transmission, using 16QAM modulation (in fading channels), has not fulfill EVM requirements at channel overlap higher than $125 \mathrm{kHz}$. In Fig. $7 \mathrm{~b}$ ), one other interesting effect is also visible. When we consider the EPA $5 \mathrm{~Hz}$ channel model (at $\mathrm{C} / \mathrm{N}=25 \mathrm{~dB}$ ) in the LTE system, then sub-frames, using 64QAM modulation, are never fulfilled to the minimal limit of EVM. This is the reason why the EVM limit for 64QAM is not marked in Fig. 7 b).

After that, we explored the dependence of the SDR ratio on the level of channel overlap of co-existing DVB-T2-Lite and LTE services. All results were obtained in both the ideal and portable fading channels and are shown in Fig. 8 a) and b). Negative values of SDR parameter present the case, when the spectral density of the TV level is higher than the level of LTE services. From these pictures it is seen that we explored possible situations which can occur at overall channel overlaps of considered services (DVB-T2-Lite vs. LTE and vice versa). Possible situations are clearly explained in the legend of Fig. 8 a) and b). For a better explanation of these results, we describe a specific example (marked by black rectangular in Fig. 8 a)). 


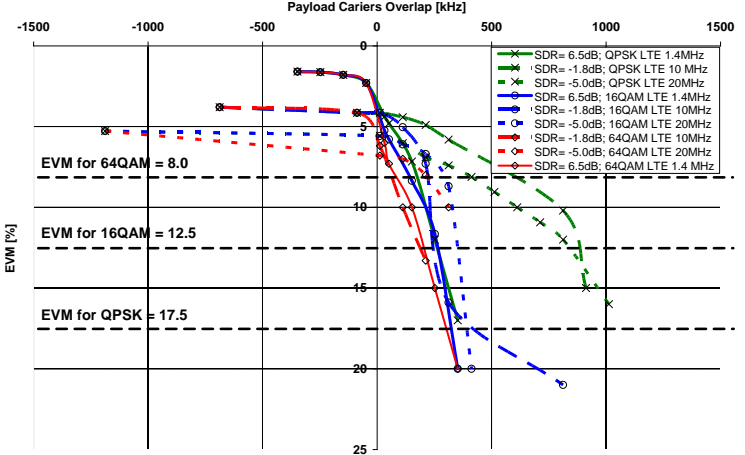

a) Ideal channel conditions.

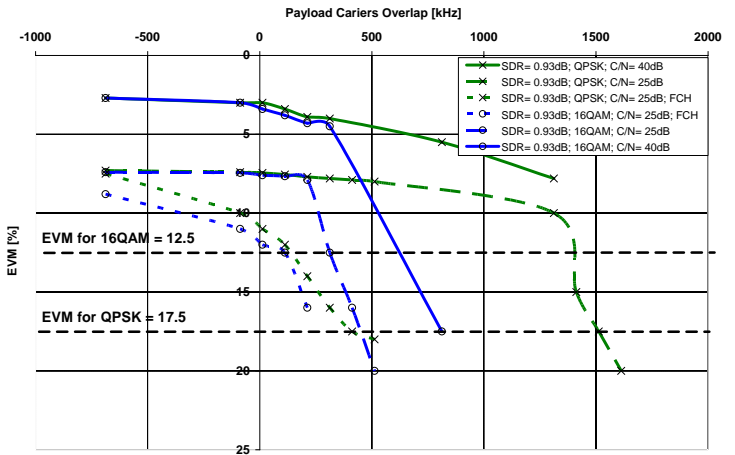

b) Gaussian and considered fading channel models.

Figure 7. EVM dependency of the QPSK, 16QAM and 64QAM modulations (using in the LTE system) on the level of frequency overlap between the DVB-T2-Lite and LTE services, working abreast in the same frequency band at ideal (a) and portable fading channel conditions (b).
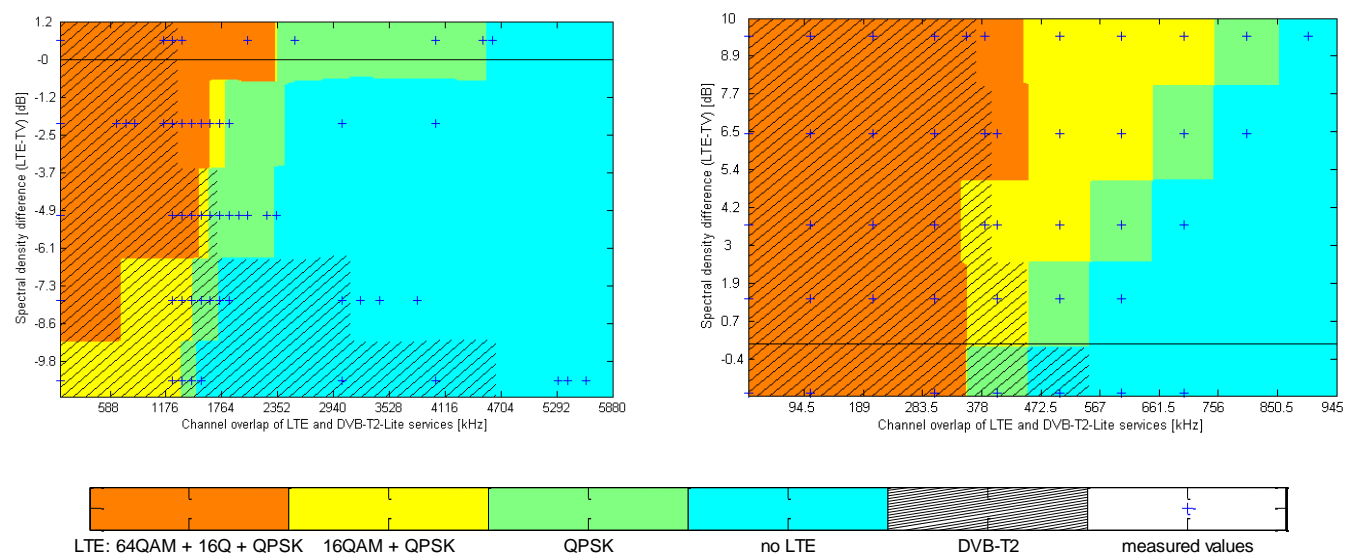

a) Ideal channel conditions ( $B_{\mathrm{LTE}}=1.4 \mathrm{MHz}$ (left) and $20 \mathrm{MHz}$ (right)).
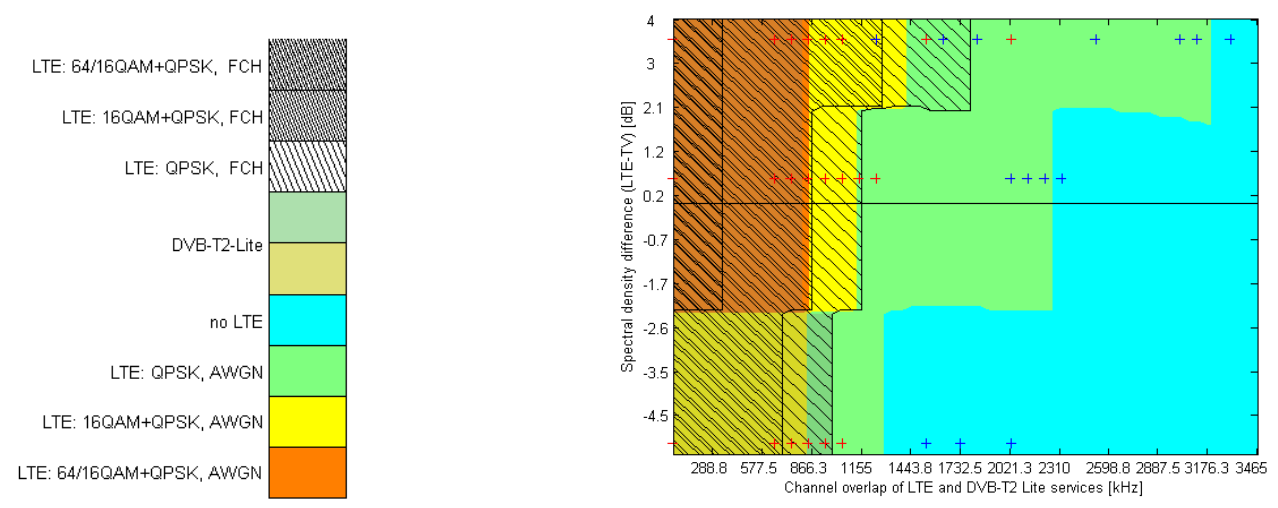

b) Gaussian and considered fading channel models $\left(B_{\mathrm{LTE}}=10 \mathrm{MHz}\right)$.

Figure 8. Graphical presentation of performance of the co-existing LTE and DVB-T2-Lite services as a dependence of SDR on the level of the channel overlap of explored services at ideal (a) and portable fading channel conditions (b). 


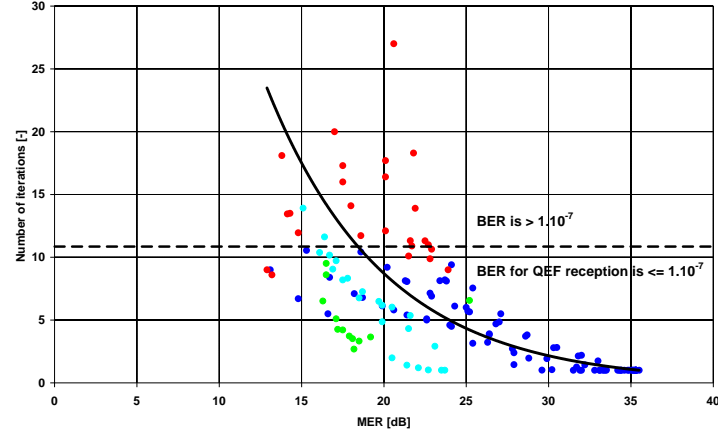

Figure 9. Dependence of the amount of repeated (number of iterations) LDPC decoding on the MER, at which the $B E R$ is less or equal to $10^{-7}$ (limit for QEF reception) at ideal and portable fading channels.

For example, we consider two fields with yellow color (see Fig. 8 (a) right, when $B_{\mathrm{LTE}}=20 \mathrm{MHz}$ ), where the spectral density of LTE is higher than the spectral density of T2-Lite. More precisely, we focus on the fields where the channel overlap is approx. from $560 \mathrm{kHz}$ to $661.5 \mathrm{kHz}$ and the spectral density differences are from $5.2 \mathrm{~dB}$ to 10 $\mathrm{dB}$, respectively (marked by black rectangular). As can be seen from the legend, in LTE system, only sub-frames using QPSK and 16QAM modulations will be received and demodulated correctly. Subframes using 64QAM modulation at these conditions can not be successfully processed. Furthermore, this field also indicates that the services of DVB-T2-Lite are completely noised (there are no hatched parts). Similar graphical representation of co-existences is achieved for the considered portable fading channels (see Fig. 8 b).

We also investigated the overall performance of DVB-T2-Lite, when it is affected by LTE services, which work in the adjacent frequency band. Our attention is focused on the dependence of the amount of repeated LDPC decoding on MER (Modulation Error Ratio). MER [7] is a measure for evaluation used to quantify the performance of a digital transmitter or receiver in a communications system using digital modulation. In the area of DVB, it is a measure of the sum of all interference effects, occurring in the transmission link. Results, obtained from our measurement, are shown in Fig. 9. Once again, the results were obtained at ideal and different portable fading channel conditions, respectively. At ideal channel conditions, the measurements were done for LTE services with different bandwidths. In the remaining channel models, the measurements were repeated for LTE with a 10 $\mathrm{MHz}$ bandwidth only.

For better evaluation, we divided the results into two parts. This division is marked in Fig. 9 by a bold dashed line. Below the dashed line is the area, where BER after LDPC decoding was less or equal to $10^{-7}$. More precisely, this area marks where the limit for QEF reception is fulfilled. At this part it can be seen that the number of needed iterations is low and the MER is high. Generally, higher MER ratios mean less unwanted interfering and noising effects in transmission/reception (dark blue dots in Fig. 9). In this part the absolute MER limit for the QEF reception is equal to $18.5 \mathrm{~dB}$ at the $\mathrm{BER}$ (after LDPC decoding) $1.10^{-7}$. This value was achieved maximally after ten decoding processes.

In the case of the aforementioned fading channels, (light green dots in Fig. 9), the MER is less, but the needed amount of decoding iterations are only slightly less than that at ideal and Gaussian (light blue dots) channel conditions. This could be caused by the equalization applied on the received DVB-T2Lite signal before LDPC decoding.

Above the dashed line (see Fig. 9) the values of the measured modulation error ratio presents the situation, when the limit for QEF operation was not fulfilled in the ideal and non-ideal transmission environments. This part represents two states. The first one is where BER after LDPC decoding is higher than $10^{-7}$, but the quality of the received signal (dots in the field near to the bold dashed line) is still good. In the second field of this part, the MER values (marked by red dots) are low (hence BER after LDPC decoding is high) and the quality of received signal is bad. At this part, the "cliff-off" effect [7] occurred many times.

Finally, snapshots of the RF spectrums of the DVB-T2-Lite and LTE services in all considered transmission scenarios are shown in Fig. 10 to. Fig. 15. Units in the ordinate are related to bandwidth of RBW filter $10 \mathrm{kHz}$. The RF spectrums at reference and non-ideal channel conditions were obtained at $\mathrm{C} / \mathrm{N}=40$ and $25 \mathrm{~dB}$, respectively. The RF spectrum of DVB-T2-Lite 
and LTE signals working in the same frequency band in reference (Gaussian) and PI and EPA 5 $\mathrm{HZ}$ portable channel models (without any coexistence) are shown in Fig. 10 and Fig. 11, respectively. The case when the T2-Lite services are highly affected by LTE ones in fading channel conditions is plotted in Fig. 12. Due to the considered channel conditions (deep fadings in the spectrums) and high interaction between both services, the quality of provided mobile services in both standards is quickly decreasing. Finally, mutual interactions between T2-Lite and LTE services in Gaussian and portable TV and mobile fading channels, when the signal level of the LTE is less or higher than T2-Lite, are plotted in Fig. 13 to Fig. 15.

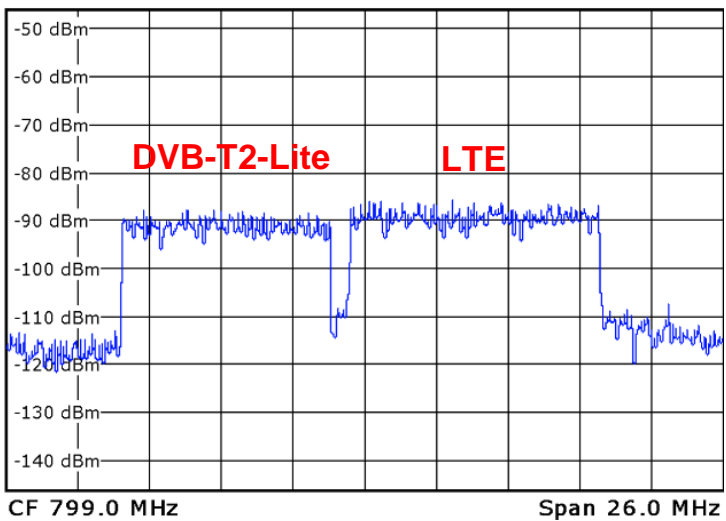

Figure 10. RF spectrum of DVB-T2-Lite and LTE services (equal signal levels) in Gaussian (reference) channel and without any co-existence.

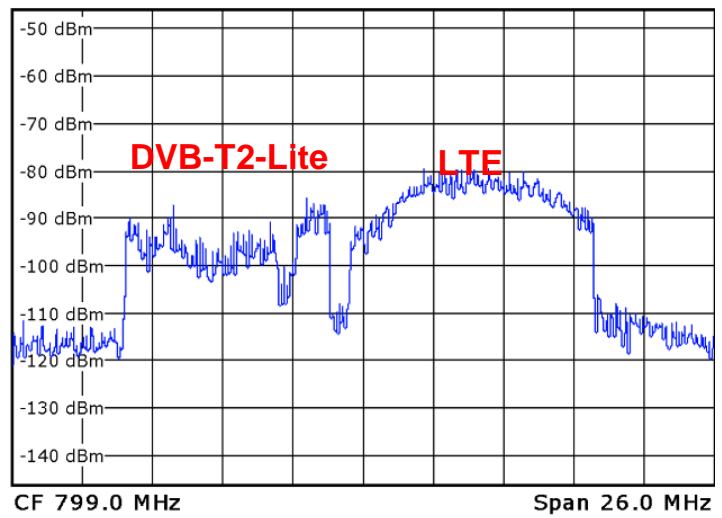

Figure 11. RF spectrum of DVB-T2-Lite and LTE services (equal signal levels) at considered portable fading channel conditions and without any co-existence.

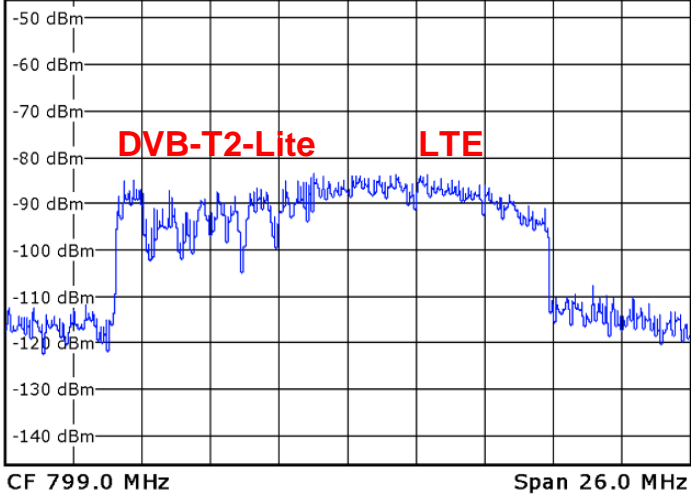

Figure 12. RF spectrum of co-existing DVB-T2 Lite and LTE services (equal signal levels) at considered portable fading channel conditions.

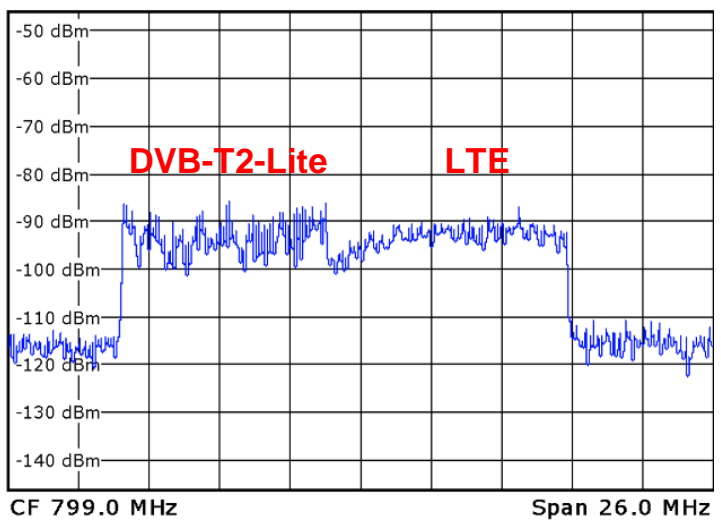

Figure 13. RF spectrum of co-existing DVB-T2-Lite and

LTE services (signal level of LTE is less than T2-Lite one) at considered portable fading channel conditions.

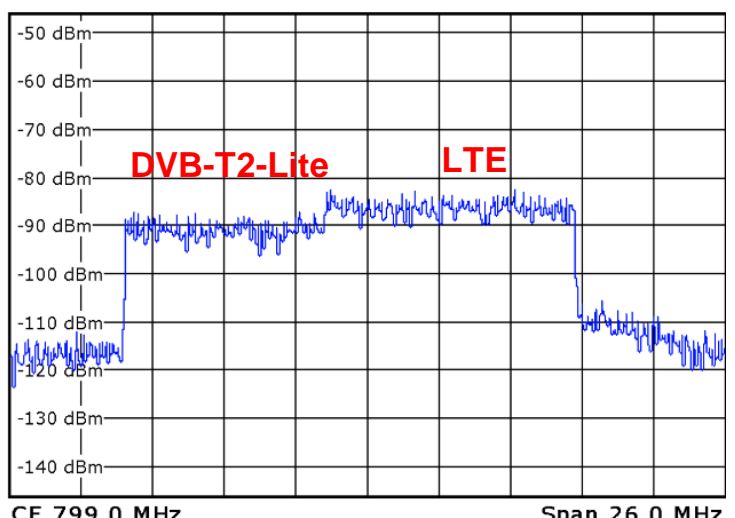

Figure 14. RF spectrum of co-existing DVB-T2-Lite and LTE services (signal level of LTE is higher than T2-Lite one) in Gaussian (reference) channel. 


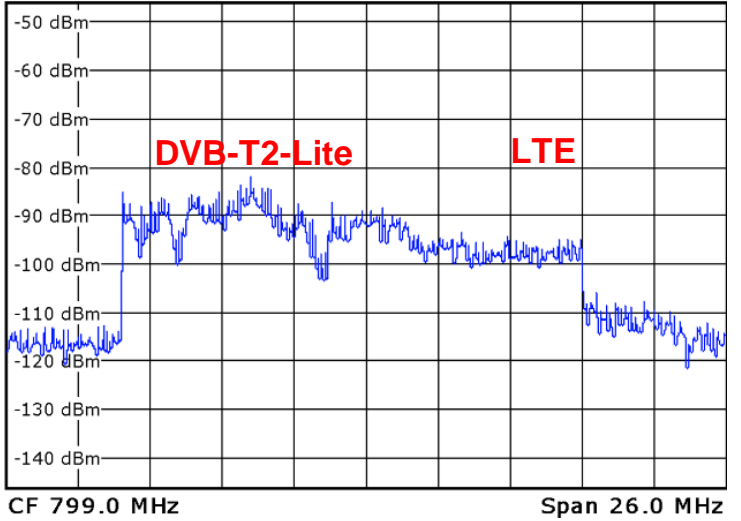

Figure 15. RF spectrum of co-existing DVB-T2-Lite and LTE services (signal level of LTE is higher than T2-Lite one) at considered portable fading channel conditions.

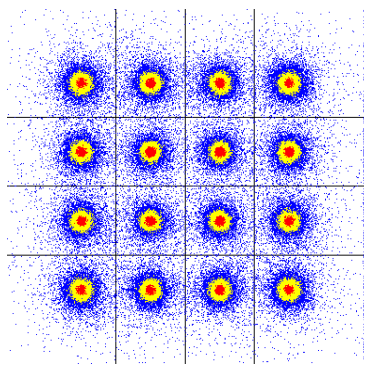

a) Non-rotated 16QAM.

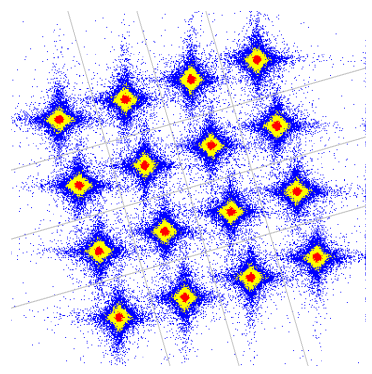

b) Rotated 16QAM.
Figure 16. Non-Rotated (a) and rotated constellation diagram (b) of 16QAM in the T2-Lite system, when the DVB-T2-Lite signal is highly affected by the LTE, (BER after LDPC decoding $\left.=2.10^{-5} ; \mathrm{MER}=19.6 \mathrm{~dB}\right)$. At this point the limit for QEF reception $\left(1.10^{-7}\right)$ is not fulfilled.

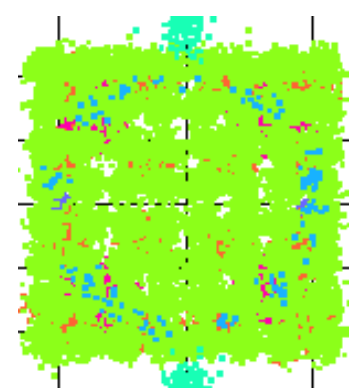

a) $A W G N+E P A 5 H z$.

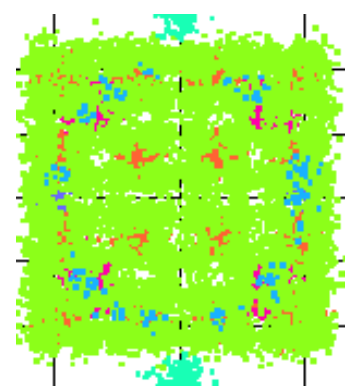

b) Co-existence with T2-Lite.
Figure 17. Constellation diagrams in the LTE system (downlink) in considered channel models (a) and at coexistence with DVB-T2-Lite services (b). There are shown all types of modulations in one constellation diagram (BPSK, QPSK, 16QAM and 64QAM).
In addition, in the case of DVB-T2-Lite, we repeated all our measurements for the case where the rotated constellation technique is used. The rotated constellation [6]-[8] in the DVB-T2 standard introduces a new technique to improve performance in channel with frequency selective fading or in the case of possible co-existence with other kinds of services. However, the obtained results proved that significant improvement in the performance of DVB-T2-Lite has not happened. Consequently, from the point of higher resistance of the DVB-T2-Lite profile against possible coexistences, the usage of rotated-constellation technique has negligible effect.

Typical results and the example or illustration of the constellation diagrams for the co-existence scenario (high channel overlap), including the used channel models, are shown in Fig. 16 and Fig 17. In Fig. 16, it is clearly seen how LTE services (operating in the same frequency band) can affect the T2-Lite services. Large distortions are visible in the non-rotated and rotated constellation diagrams. Figure 17 shows all types of modulations used in the LTE system and their distortions for downlink in one common constellation diagram.

\section{Conclusions}

In this paper the co-existence of the DVB-T2-Lite and LTE mobile services, operating in the same frequency band, was explored and measured in ideal and portable fading channel models. For this purpose (see Fig. 5), an appropriate measurement workplace was realized (see Fig. 6).

Firstly, the influence of channel overlap of coexisting services (DVB-T2-Lite and LTE) on LTE services was explored in ideal and portable fading channel models, respectively. For evaluating this influence we used the EVM parameter, related to modulations of LTE. The results in the considered fading channel models were worse, which is mainly caused by their features (higher path delay and time varying conditions). This was mainly true for sub-frames used 64QAM modulation, which at critical frequency overlap were quickly corrupted.

Secondly, we focused on deeper analysis of the co-existence scenarios such as dependence of SDR on the level of the frequency overlap. More precisely, we explored scenarios where both or 
only one mobile system worked without significant errors. From the results which were obtained in fading channels (see Fig. 8) it can be seen that at $\mathrm{BLTE}=10 \mathrm{MHz}$ the DVB-T2-Lite services are corrupted already at less frequency overlap. Overall from these results it can be seen that the decreasing performance of DVB-T2-Lite is highly depending on the power of the LTE signal and its channel bandwidth.

Finally, we investigated the performance of the DVB-T2-Lite system. We explored the dependence of the iteration number of repeated LDPC decoding on MER needed for achieving QEF operation.

This work will continue by finishing and improving the proposed method for measuring interactions between mobile DVB-T/H/T2 and LTE services in different transmission scenarios [28], [29], [34][37]. Moreover, we also consider extending our research with real filed measurements.

\section{Acknowledgments}

This paper was supported by the CATRENE EU project no. CA116 and MEYS of the Czech Republic project no. LF14033, national project no. LD12005 and no. CZ.1.07/2.3.00/20.0007 and finally by the BUT project no. FEKT-S-14-2177. Described research was performed in laboratories supported by the SIX project; no. CZ.1.05/2.1.00/03.0072, the operational program Research and Development for Innovation.

\section{References}

[1] A. Guidotti et. al., "Coexistence and mutual interference between mobile and broadcasting systems," in Proc. of the IEEE $73^{\text {rd }}$ International Conference Vehicular Technology VTC Spring, Budapest (Hungary), 2011, pp. 1-5.

[2] T. Yoshihisa, S. Nishio, "A Division-Based Broadcasting Method Considering Channel Bandwidths for NVoD Services," IEEE Transaction of Broadcasting, vol. 59, no. 1, pp. 62-71, 2013.

[3] J. A. Esquerra-Soto et. al., "Performance Analysis of 3G+ Cellular Technologies with Mobile Clients," Journal of Applied Research and Technology, vol. 10, no. 2, pp. 227-247, 2012.

[4] Coexistence Digital TV and LTE (Application Note 1MA176_e3), Rohde \& Schwarz, 2012, pp. 1-34.

[5] A. Aloisi et. al., "A simulation study of broadband mobile systems interference on DVB-T systems operating in the UHF frequency band," in 2011 Future Network \& Mobile Summit, Warsaw (Poland), 2011, pp. 1-8.

[6] EN 302755 V1.2.1. Digital Video Broadcasting (DVB); Frame structure channel coding and modulation for a second generation digital terrestrial television broadcasting system (DVB-T2), European Standard ETSI, 2011, pp. 1-188.

[7] TS 102831 V1.2.1. Digital Video Broadcasting (DVB); Implementation guidelines for a second generation digital terrestrial television broadcasting system (DVB-T2), Technical Specification ETSI, 2012, pp. 1-244.

[8] L. Vangelista et. al., "Key Technologies for NextGeneration Terrestrial Digital Television Standard DVBT2," IEEE Communications Magazine, vol. 47, no. 10, pp. 146-153, 2013.

[9] 3GPP TS 36.101 V8.8.0. $3^{\text {rd }}$ Generation Partnership Project; Technical Specification Group Radio Access Network; Evolved Universal Terrestrial Radio Access (EUTRA); User Equipment (UE) radio transmission and reception (Release 8), Technical Specification 3GPP, 2009, pp. 1-160.

[10] E. Dahlman et. al., "4G: LTE/LTE-Advanced for Mobile Broadband ( $1^{\text {st }}$ edition)", United Kingdom: Academic Press, 2011, pp. 1-455.

[11] D. Astely et. al., "LTE: the evolution of mobile broadband," IEEE Communications Magazine, vol. 47, no. 4, pp. 44-51, 2009. 
[12] J. Miulka, S. Hanus, "Bluetooth and IEEE $802.11 \mathrm{~b} / \mathrm{g}$ Coexistence Simulation," Radioengineering, vol. 17, no. 3, pp. 66-73, 2008.

[13] F. Gleissner, S. Hanus, "Co-channel and Adjacent Channel Interference Measurement of UMTS and GSM/EDGE Systems in $900 \mathrm{MHz}$ Radio Band," Radioengineering, vol. 17, no. 3, pp. 74-80, 2008.

[14] S. Kumar et. al., "Throughput Analysis of Primary and Secondary Networks in a Shared IEEE 802.11 System," IEEE Transactions on Wireless Communications, vol. 12, no. 3, pp. 1006-1017, 2013.

[15] L. Klozar et. al., "Effect of Co-Existence Interferences on QoS of HSPAWDCMA Mobile Networks," in Proc. of the $23^{\text {rd }}$ International Conference Radioelektronika 2013, Pardubice (Czech Republic), 2013, pp. 243-246.

[16] B. Han et. al., "Investigation of Interference Margin for the Co-existence of Macrocell and Femtocell in Orthogonal Frequency Division Multiple Access Systems," IEEE Systems Journal, vol. 7, no. 1, pp. 59-67, 2013.

[17] Z. Yushu et. al., "Co-existence analysis of LTE and WIMAX in uplink," in Proc. of the IEEE 6th Intern. Conf. on Wireless Communications, Networking and Mobile Computing, Chengdu (China), 2010, pp. 1-4.

[18] A. Lourerio et. al., "Interference analysis on UMTS2100 co-existence with GSM-1900," in Proc. of the IEEE $74^{\text {th }}$ International Conference Vehicular Technology VTC Fall 2011, San Francisco (USA), 2011, pp. 1-4.

[19] K. Ruttik et. al., "Modeling of the Secondary System's Generated Interference and Studying of its Impact on the Secondary System Design," Radioengineering, vol. 19, no. 4, pp. 488-493, 2010.

[20] K. Šakić et. al., "Cross-border interference between broadcasting and mobile services," in Proc. of the IEEE $51^{\text {st }}$ International Symposium ELMAR 2009, Zadar (Croatia), 2009, pp. 229-232.

[21] T. Kratochvil, V. Ricny, "Simulated testing of the interferences influence on the DVB-T signal," in Proc. of the $19^{\text {th }}$ International Conference Radioelektronika 2009, Bratislava (Slovakia), 2009, pp. 107-110.

[22] K. Šakić, S. Grgić., "The Influence of the LTE System on DVB-T Reception," in Proc. of the IEEE $52^{\text {nd }}$ International Symposium ELMAR 2010, Zadar (Croatia), 2010, pp. 235-238.

[23] I. Cho, "Analysis on Interference Impact of LTE on DTV," in T.-H. Kim et. al. (eds), Multimedia, Computer Graphics and Broadcasting, Part I, Berlin (Germany): Springer, 2012, pp. 344-350.
[24] Z. A. Shamsan, "LTE-Advanced Compatibility with Digital Broadcasting Receiver at $800 \mathrm{MHz}$," in Proc. of the IEEE $2^{\text {nd }}$ Electronics, Communications and Photonics Conference SIEPC 2013, Riyadh (Saudi Arabia), 2013, pp. 1-4.

[25] L. Polak et. al., "Measuring of the interactions between mobile and DVB-T broadcasting networks," in Proc. of the $22^{\text {nd }}$ Intern. Conference Radioelektronika 2012, Brno (Czech Republic), 2012, pp. 243-246.

[26] L. Polak et. al., "Influence of Mobile Network Interfering Products on DVB-T/H Broadcasting Services," in Proc. of the IEEE $5^{\text {th }}$ International Conference Wireless Days 2012, Dublin (Ireland), 2012, pp. 1-5.

[27] L. Polak et. al., "Exploring and measuring the coexistence between LTE and DVB-T2-Lite services," in Proc. of the $36^{\text {th }}$ Intern. Conference on Telecommunications and Signal Processing (TSP), Rome (Italy), 2013, pp. 316-320.

[28] L. Polak, T. Kratochvil, "DVB-H and DVB-SH-A Performance and Evaluation of Transmission in Fading Channels," in Proc. of the $34^{\text {th }}$ Intern. Conference on Telecommunications and Signal Processing (TSP), Budapest (Hungary), 2011, pp. 549-553.

[29] L. Polak, T. Kratochvil, "Simulation and Measurement of the Transmission Distortions of the Digital Television DVB-T/H Part 3: Transmission in Fading Channels," Radioengineering, vol. 19, no. 4, pp. 703-711, 2010.

[30] TS 136101 V8.15.0. LTE; Evolved Universal Terrestrial Radio Access (E-UTRA); User Equipment (UE) radio transmission and reception, Technical Specification ETSI, 2011, pp. 1-171.

[31] J. A. Del Peral-Rosado et. al., "Joint channel and time delay estimation for LTE positioning reference signals," in Proc. of the $6^{\text {th }}$ ESA Workshop on Satellite Navigation Technologies and European Workshop on GNSS Signals and Signal Processing 2012, Noordwijk (Netherlands), 2012, pp. 1-5.

[32] Z, Raida., et. al. "Center of Sensor, Information and Communication Systems (SIX): Full Operation Starting," Radioengineering, vol. 22, no. 4, pp. 1108-1116, 2013.

[33] IEC 62216-1 ( $1^{\text {st }}$ edition). Digital terrestrial television receivers for the DVB-T system - Part 1: Baseline receiver specification, International Standard, 2001, pp. 1-118.

[34] I. Cho et. al., "Study on Coexistence between Long Term Evolution and Digital Broadcasting services" International Journal of Advanced Science and Technology, vol. 39, pp. 75-92, 2012. 
[35] Y. L. Lee et. al., "Improved Detection Performance of Cognitive Radio Networks in AWGN and Rayleigh Fading Environments," Journal of Applied Research and Technology, vol. 11, no. 3, pp. 437-446, 2013.

[36] A. Tekovic et. al., "LTE downlink system performance measurement with intersystem interference caused by DVB-T signal," in Proc. of the IEEE 54th International Symposium ELMAR 2012, Zadar (Croatia), 2012, pp. 255-258.

[37] T. Kratochvil, R. Stukavec, "DVB-T Digital Terrestrial Television Transmission over Fading Channels," Radioengineering, vol. 17, no. 3, pp. 96-102, 2008. 